\title{
Du service public au service citoyen
}

La Scic, un statut adapté à cette ambition

From public services to citizen services

How the SCIC form suits this ambition

Desde el servicio público hasta el servicio ciudadano

La SCIC, un estatuto adaptado a esta ambición

\section{Yves-Alain Liénard}

Numéro 340, avril 2016

Scic

SCIC

URI : https://id.erudit.org/iderudit/1037403ar

DOI : https://doi.org/10.7202/1037403ar

Aller au sommaire du numéro

Éditeur(s)

Association Recma

ISSN

1626-1682 (imprimé)

2261-2599 (numérique)

Découvrir la revue

Citer cet article

Liénard, Y.-A. (2016). Du service public au service citoyen : la Scic, un statut adapté à cette ambition. Revue internationale de l'économie sociale, (340), 65-76. https://doi.org/10.7202/1037403ar
Résumé de l'article

En France, le service public a été porté à l'idéal, mais, dans les faits, force est de constater qu'il doit évoluer. Le statut Scic pourrait-il permettre de le renouveler ? L'une des mesures de l'utilité sociale des entreprises se fait à l'aune de ses effets sur la société. Le statut Scic permet à certaines entreprises d'avoir un impact large sur les citoyens. L'expérience des Scic du réseau Enercoop donne à voir que son utilité tend à dépasser le seul intérêt de ses membres et bénéficiaires.

Dès 1984, en raison de la crise de l'Etat-providence, Pierre Rosanvallon envisageait la possibilité d'expérimenter des « auto-services collectifs » en parallèle ou substitution aux services publics. La Scic peut-elle constituer un outil efficace pour passer, dans certains cas, du service public au service citoyen? 


\title{
DU SERVICE PUBLIC \\ AU SERVICE CITOYEN
}

\section{LA SCIC, UN STATUT ADAPTÉ À CETTE AMBITION}

\author{
par Yves-Alain Liénard*
}

\author{
* Président de la Scic Enercoop \\ Languedoc-Roussillon.
}

En France, le service public a été portéà l'idéal, mais, dans les faits, force est de constater qu'il doit évoluer. Le statut Scic pourrait-il permettre de le renouveler? L'une des mesures de l'utilité sociale des entreprises se fait à l'aune de ses effets sur la société. Le statut Scic permet à certaines entreprises d'avoir un impact large sur les citoyens. L'expérience des Scic du réseau Enercoop donne à voir que son utilité tend à dépasser le seul intérêt de ses membres et bénéficiaires. Dès 1984, en raison de la crise de l'Etat-providence, Pierre Rosanvallon envisageait la possibilité d'expérimenter des "auto-services collectifs" en parallèle ou substitution aux services publics. La Scic peut-elle constituer un outil efficace pour passer, dans certains cas, du service public au service citoyen?

\section{From public services to citizen services: How the SCIC form suits this ambition}

Public services in France are idealized but the reality forces us to recognise that they have to change. Would the SCIC form enable remodelling public services? One measure of an enterprise's social utility is its effect on society. The SCIC form allows certain businesses to have a wide impact on the citizens. The experience of the SCICs in the Enercoop group shows that its usefulness is greater than just the interests of its members and beneficiaries. Already in 1984, with the crisis in the welfare state, Pierre Rosanvallon envisaged the possibility of experimenting with self-managed collective services working in tandem with public services or replacing them. Can the SCIC form be an effective tool for transitioning from public services to citizen services in certain cases?

\section{Desde el servicio público hasta el servicio ciudadano. La SCIC, un estatuto adaptado a esta ambición}

En Francia, el servicio público se eleva al rango de ideal, pero en los hechos se constata que debe evolucionar. ¿Podría el estatuto Scic (sociedad cooperativa de interés colectivo) permitir de renovarlo? La utilidad social de las empresas se mide, entre otras cosas, por sus efectos sobre la sociedad. Con el estatuto Scic, ciertas empresas pueden tener un impacto amplio sobre los ciudadanos. El caso de las Scic de la red Enercoop demuestra que su utilidad tiende a superar el interés único de sus miembros y beneficiarios.

Desde 1984, debido a la crisis del Estado-providencia, Pierre Rosanvallon había considerado la posibilidad de experimentar "auto-servicios colectivos", en paralelo o sustitución de los servicios públicos. ¿Puede la Scic constituir un instrumento eficaz para pasar, en algunos casos, del servicio público al servicio ciudadano? 
Salariés, usagers, producteurs, collectivités, partenaires, bénévoles... constituent des catégories de personnes physiques ou morales qui peuvent être parties prenantes et donc former les associés des sociétés coopératives d'intérêt collectif (Scic).

Par l'origine de ses membres, les Scic peuvent représenter la société dans toute sa diversité, ce qui souligne à la fois leur originalité et leur impact. De plus, certaines Scic peuvent proposer des services nécessaires à la vie quotidienne et sociale de tous et leur forme coopérative facilite, voire oblige à un traitement égalitaire, équitable et tourné vers l’intérêt général, non sans lien avec la notion de service public ${ }^{(1)}$.

Dans cette période de repli sur soi et de marchandisation galopante au cours de laquelle le concept même de service public est malmené, voire contesté, la Scic, dans certains cas, ne serait-elle

(1) Lire article « Servicepublic», dictionnaire Le Nouveau Petit Robert, Paris, Le Robert, 1993. pas une réponse possible pour orienter les choix vers la société dans laquelle nous voulons vivre?

Nous nous attacherons à envisager la notion de service public et sa spécificité française, avant de comprendre pourquoi cet idéal bienveillant a pu être dévoyé. Nous interrogerons les liens entre intérêt collectif et intérêt général, puis nous examinerons comment la Scic peut recréer un modèle citoyen et démocratique de refondation de ces services. Nous nous appuierons pour cela sur l'exemple et l'expérience d'Enercoop, puis nous étendrons la réflexion aux domaines de l'éducation ou la santé.

\section{Services publics}

\section{Rappel historique}

L'historique des services publics révèle qu'ils ont d'abord été institués comme garants de l'intégrité du royaume et de l'expression de la puissance de l'Etat, quel que soit son régime. Xavier Bezançon (1995) différencie les services publics d'Etat en trois catégories principales: les services publics régaliens (justice [armée, police, tribunaux], monnaie, impôts) et les services publics locaux; les services urbains de proximité (eau, éclairage); les secours essentiels (contre le feu et les hôpitaux). Ceux-ci relèvent soit d'un service nécessaire et vital, qui correspond à une menace et à une obligation de moyens, soit d'un service opportun qui facilite et agrémente la vie publique et répond à une obligation de résultat. Avec la Révolution et la Déclaration des droits de l'homme s'instaure la notion d'« intérêt général ", mais c'est à la fin du XIX ${ }^{\mathrm{e}}$ siècle que s'opère le basculement de la notion de puissance d'Etat, entité extérieure et supérieure, vers l'idée de l'interventionnisme, de la solidarité et la création de la notion d'« Etat-providence », à la suite des travaux de Léon Bourgeois et de Charles Gide.

\section{Spécificités françaises}

Durant toute la première moitié $\mathrm{du} \mathrm{XX}^{\mathrm{e}}$ siècle, ce glissement s'est opéré pour aboutir à cette notion du service public «à la française ", telle que la définit le dictionnaire: "Fonction de l'Etat pour la satisfaction des besoins de la collectivité nationale. »

Les fondements du service public français sont basés sur la continuité, l'égalité et la mutabilité: pérenne, identique pour tous et capable d'évoluer. 
On demande au fonctionnaire d'être compétent (assuré par le concours), neutre, de ne privilégier personne (ni corruption ni pression, car son emploi est garanti). L'image de l'agent de la fonction publique a souvent été moquée et a pu ne pas toujours correspondre à cette idée affirmée du service public. C’est toutefois bien vers cet idéal et adossés sur ces principes qu'ont été construits les services publics en France.

Le service public français a contribué à la formation de "l'Etat-providence » par opposition à « l'Etat-gendarme » défendu par les libéraux et la doxa anglosaxonne. Est-ce à l'Etat de réduire les inégalités, en assurant une meilleure répartition de la richesse produite, ou au marché et à l'initiative privée de réguler les rapports sociaux, l'Etat ne faisant qu'encadrer le marché par une législation sociale minimale? Notre intention n'ambitionne pas d'être exhaustif sur le sujet, mais de souligner ce que véhicule cette notion de service public et de rappeler qu'il existe plusieurs points de vue et plusieurs approches autour de cette notion. En effet, au cours du XX ${ }^{\mathrm{e}}$ siècle, comme l'a écrit Jacques Chevallier, "le service public a été érigé en France à la hauteur d'un véritable mythe, c'est-à-dire une de ces images fondatrices, polarisant les croyances et condensant les affects, sur lesquelles prend appui l'identité collective. [...] Le service public est un opérateuridéologique: il sculptele mythe d'un Etat généreux, bienveillant, uniquement soucieux du bien-être de tous " (Chevallier, 2012 [1987]).

\section{Le temps des remises en cause}

Pourtant, dans les deux dernières décennies du $\mathrm{XX}^{\mathrm{e}}$ siècle, les principes et le sens de l'action publique furent interrogés. En 1994, une publication d'André Rossinot, ministre de la Fonction publique ${ }^{(2)}$, posait

(2) Ministre de la Fonction publique du 30 mars 1993 au 11 mai 1995. explicitement la question: "Une fonction publique est-elle encore utile à l'aube duXXI siècle?» (Rossinot, 1994). Dans sa conclusion, l'auteur affirmait que, pour ne pas disparaître, la fonction publique devait s'adapter à son temps.

Avec la chute du mur de Berlin et l'écroulement du système soviétique, puis avec les mutations du modèle capitaliste liées à la financiarisation et à l'émergence de l'économie numérique, les valeurs du libéralisme économique, ancrées sur une individualisation des comportements, ont gagné en puissance. Ces valeurs, vecteurs de délitements sociaux, ont contaminé toute la société et remis subséquemment en question la notion même de services publics.

Ces derniers ont été attaqués sur tous les fronts: rentabilité, performance individuelle, situation de monopole, frein à l'innovation, dépenses indues. Conjuguée à la forte concentration des lieux de décision - "L'Etat, c'est l'administration centrale, soit trois pour cent des fonctionnaires de l'Etat: trois pour cent, mais beaucoup de pouvoir » (Rossinot, 1994)-, la société civile elle-même a évolué dans son rapport au service public en mettant en question son représentant: le fonctionnaire. Dans les faits, l'usager est devenu un client et cette mutation sémantique elle-même n'est pas sans conséquence. L'usager fait... usage, il subit une action commune ou y participe, selon les points de vue. Le client a des droits, s'il est mécontent, il change de prestataire et rien ne doit restreindre sa liberté de choix, de telle sorte 
qu'il ne se soucie pas de réformer ou d'améliorer: en consommateur sûr de ses droits, il ignore. Cette évolution des comportements suggère un changement de paradigme du concept de service public, continûment à l'œuvre depuis l'après-guerre.

En résonance avec la vision néo-libérale, qui vise à la « démolition du service public », Dardot et Laval (2014) expliquent que le capitalisme financier entreprend aujourd'hui un mode d'accumulation par dépossession. Après avoir accumulé en " exploitant » (les ressources et les hommes), le nouvel âge du capitalisme vise une marchandisation totale en s'accaparant le commun: l'eau, les routes, l'école, demain l'air et le paysage. Le démantèlement des services publics est l'un des corollaires répondant à cette logique marchande guidée par le profit et une rentabilité maximale de court terme.

"Dans un futur perçu comme la simple suite des menaces qui pèsent sur l'humanité, la gestion de crise devient le rapport exclusif à l'avenir et la volonté de maîtrise s'accompagne d'une prolifération des outils de contrôle, qui prend le pas sur l'idéal politique d'un gouvernement tournévers l'émancipation de l'homme» (Cohen, 2008). Cette assertion constate que le rapport à l'avenir est devenu anxiogène et vecteur non pas d'émancipation, mais d'un contrôle accru.

Face à cette vision de l'avenir projetée comme une suite de menaces, où les outils de contrôle sont technologiques, réglés par des algorithmes, et soustendue par une fascination pour les nombres, d'autres approches alternatives se construisent.

Comme nous l'avons déjà indiqué, dès le milieu des années 80, Pierre Rosanvallon (1984) affirmait qu'il était " urgent de sortir de cette alternative étatisation/privatisation [...]. Le "qui doit payer les services collectifs?" fait oublierle "qu'est-ce qu'un service collectif?” ». Plus de trente ans plus tard, ces questions demeurent de pleine actualité, mais on perçoit que nous sommes à la croisée des chemins. Si la question du démantèlement des services publics alimente les débats, une mutation de cette offre publique de service pourrait passer par une expérimentation refondatrice au sein d'entreprises qui allient initiative privée et gestion collective.

\section{Intérêt collectif versus intérêt général}

\section{L’intérêt général}

L'intérêt général peut être envisagé selon deux approches: l'une utilitariste, l'autre interventionniste. Soit il est seulement la somme des intérêts individuels, et il pourrait en ce sens exprimer un consensus, soit il doit dépasser ceux-ci et être d'utilité publique. Selon les termes de cette alternative, il y a le risque de produire soit un assemblage d'" égoïstes », soit un groupe d'" assistés ». Cette vision forcée et réductrice révèle l'insatisfaction que peuvent induire ces deux visions de l’intérêt général. Au nom de celui-ci, il y a le risque d'une forte concentration des lieux de décision et d'un décrochage avec les attentes du plus grand nombre. Faute de trouver le juste équilibre entre écoute et prise de décision, mais à n'écouter que la vox populi, il ne faut pas omettre le risque de la démagogie ou du populisme. 


\section{L'intérêt collectif}

En France, l'intérêt collectif est trop souvent suspecté de favoriser le communautarisme ou un corporatisme latent. Pourtant, ignorer des groupes localisés sur un territoire ou des communautés d'intérêt revient à bafouer la possibilité d'expertise de ces mêmes groupes.

Nous en trouvons un exemple à la fin des années 80. La notion de santé communautaire a alors fait irruption dans le domaine de la toxicomanie. Depuis 1972, la vente libre des seringues était interdite. Ainsi, les usagers se prêtaient ou réutilisaient celles-ci, favorisant l'expansion du sida. Faisant valoir une notion d'intérêt général, contre des avis très majoritairement opposés, Michèle Barzach, ministre de la Santé, autorisa en 1987 la vente de seringues au nom de la lutte contre la diffusion du VIH et pour préserver la sécurité publique et celle des officines. La " communauté » des toxicomanes comprit alors très rapidement que son intérêt collectif consistait à s'approprier cette possibilité. Devant la méconnaissance de ces nouveaux usages et la désinformation, des associations d'autosupport se constituèrent pour diffuser des messages de prévention et d'accompagnement. En quelques mois, la prévalence du virus par voie intraveineuse chuta spectaculairement.

Cet exemple souligne que l'association de l'intérêt général et de l'intérêt collectif, ayant mis en contact des acteurs différents, où

(3) Le commissaire du gouvernement Delmas-Marsalet dans ses conclusions relatives à l'arrêt du 30 novembre 1973 (association Saint-Luc, clinique du Sacré-Cœur, $\mathrm{n}^{\circ} 85586-85598$ ). Texte repris dans la circulaire adressée aux préfets en avril 2002 concernant les conditions de l'agrément des sociétés coopératives d'intérêt collectif, Social-sante.gouv. fr/fichiers/bo/2002/02-24/ a0242176.htm.

(4) La foncière est une entreprise d'investissement solidaire ouverte aux citoyens. Elle permet à chacun de placer son épargne dans un projet à haute valeur sociale et écologique. Le capital accumulé sert à acheter des fermes pour y implanter des activités agrirurales diversifiées. La foncière loue ces fermes à des paysans engagés dans une agriculture de proximité, biologique et à taille humaine. Source: Terredeliens.org. chacun est resté dans son domaine, peut être bénéfique pour tous.

\section{L'économie sociale et solidaire}

L'expansion de l'économie sociale et solidaire (ESS) pourrait être une réponse, une voie sinon médiane, du moins différente. La singularité de l'ESS est inscrite dans la loi : gouvernance participative, où l'expression de chacun n'est pas liée à son apport en capital, répartition des bénéfices majoritairement consacrés au maintien et au développement de l'entreprise, caractère d'utilité sociale. Trop longtemps cantonné au seul volet social de l'aide aux démunis et à l'accompagnement de la précarité, un arrêt de 1973 stipule pourtant : "Le caractère d'utilité sociale d'une institution ne découle pas du secteur dans lequel elle exerce son activité, mais bien des conditions dans lesquelles elle l'exerce ${ }^{(3)}$."

Ainsi, la foncière Terre de liens ${ }^{(4)}$ collecte des fonds pour acheter des terres agricoles et les mettre à disposition ou les louer à des paysans, afin de garantir leur accès à la terre. Ses statuts garantissent des "pratiques agricoles respectueuses de l'environnement à très long terme ». Il y a bien une utilité sociale ayant un impact direct pour la personne installée, comme pour la société dans son entier et principalement pour les personnes qui résident à proximité. A l'inverse de ces pratiques, d'autres entreprises d'investissement achètent des terres agricoles dans le seul but spéculatif. La spécificité des structures de l'ESS est d'envisager la réussite économique non comme une finalité, mais comme un moyen au service d'un projet. 


\section{Services citoyens, le champ des possibles}

\section{Le statut Scic}

Une fédération syndicale écrivait il y a peu: «Il y a urgence à réinventer un système démocratique s'appuyant sur la participation des citoyens, des partenaires sociaux et des acteurs clés du système» (Becker et al., 2015). Ce système existe, ce sont les Scic: les prises de décision y sont démocratiques et diverses parties prenantes sont associées en son sein.

Ce statut coopératif récent est l'aboutissement d'une réflexion sur la création d'entreprises ayant un but social, dont l'activité s'exerce dans le secteur marchand. Néanmoins, par rapport aux coopératives, il n'est pas obligatoire d'être membre associé pour acheter l'un des biens ou services produits et la pratique de la " ristourne » en est absente. Le statut Scic ne limite ni la taille ni le domaine de l'objet de la société. Certaines peuvent vouloir rassembler par le biais du multisociétariat toutes les parties prenantes d'un territoire autour d'un projet précis (par exemple la Scic Orchis Eaulogie, à Montpellier, bureau d'études axé sur le développement durable et spécialisé dans la gestion de l'eau), d'autres proposer un autre modèle, dans l'esprit du service public: "L'accès à l'énergie est un besoin essentiel. Le modèle français ne permet ni de le satisfaire correctement, ni de remplir les missions de service public de l'énergie [...].Enercoop se donne pour mission d'offrir un service énergétique complet ${ }^{(5)}$. » Un seul statut, mais de multiples possibilités qui trouveront souvent leur expression et leur particularité dès la rédaction des statuts.

(5) Enercoop, 2015, extrait de la charte-réseau Enercoop. En 1926, Bernard Lavergne proposait le modèle de la régie coopérative, qui permettait de "découvrir une formule de gestion qui unisse les avantages sociaux de l'entreprise d'Etat à la valeur commerciale et technique de l'entreprise privée »(Lavergne, 1926). Néanmoins, la régie coopérative qu'il décrivait alors se différenciait de la Scic, car, répondant aux principes coopératifs, il n’envisageait pas de distribuer les produits à des non-coopérateurs; en revanche, il s'en éloignait en formulant des propositions sur la répartition des votes et la « ristourne ». Dans une Scic, ni « ristourne » ni augmentation de la valeur de la part sociale. Dans le cadre de la régie coopérative, Bernard Lavergne pensait vraisemblable que "les régies dans bien des cas [...] préféreront abaisser leurs tarifs à l'avenir plutôt que de rendre une ristourne à leurs consommateurs anciens: chaque fois que la régie a un grand nombre de clients, cette façon de procéder est très avantageuse » (Lavergne, 1926, p. 207). De même, il proposait dans sa description une pondération des votes, car, si des collectivités aussi disparates qu'une capitale et un petit village étaient sociétaires, il ne serait pas équitable de leur donner le même poids dans les décisions. Au sein de la Scic, chaque personne morale ou physique ne dispose que d'une voix, mais par le biais des collèges de vote les statuts peuvent prévoir une pondération de ceux-ci.

Il s'agit donc bien de réinventer un système, même si celui-ci emprunte aux modèles antérieurs. En outre, la création du statut Scic peut, dans certains cas, s'appliquer au renouvellement des services publics. Reste à rechercher "quelles industries et quels services publics se prêtent à la coopératisation (sic) envisagée sous la forme des régies coopératives ( (Lavergne, 1926, p. 50) ou, pour le dire en termes plus actuels, sous la forme de Scic. 


\section{L'exemple d'Enercoop}

\section{LA GENÈSE}

L’ouverture du marché de l'électricité et la réflexion antinucléaire ont suscité des débats qui ont conduit plusieurs acteurs à proposer une alternative. Enercoop est née de cette dynamique citoyenne et s'est donné pour objet «la vente et l'achat d'énergie produite à partir de sources d'énergies renouvelables [...], ainsi que la fourniture de services énergétiques dans l'objectif de diminuer les consommations d'énergie et d'augmenter la part des énergies renouvelables dans le bilan énergétique national ${ }^{(6)}$ ».

(6) Enercoop, article 3 des Outre la promotion des énergies renouvelables et la volonté de statuts. proposer un modèle industriel différent, Enercoop s'emploie à impliquer directement le consommateur final. Le modèle de la Scic s'est donc imposé naturellement. Le point crucial réside dans la diversité des parties prenantes. Ainsi se retrouvent autour de la table de la gouvernance des producteurs, des salariés et des consommateurs qui, au sein de la chaîne du produit ou du service, peuvent avoir des intérêts divergents. Mais la discussion autour des finalités, nécessaire pour assurer la pérennité de l'entreprise, peut aboutir à une prise de conscience de leur interdépendance. A ces catégories « de base ", Enercoop a ajouté des personnes morales (partenaires, collectivités, financeurs), qui peuvent contribuer à cimenter le projet dans sa pérennité.

Nous verrons que, dans le sillage de cette coopérative, neuf autres ont été créées, avec des statuts quasiment similaires. Avec le temps, nous sommes passés de six à onze catégories. Cette expansion a été volontaire et permet de préciser tous les points d'entrée possibles au sociétariat.

Comme dans le cadre de la régie coopérative de Bernard Lavergne, une pondération des votes par le biais des collèges a été mise en place. Il ne s'agit pas ici de refléter un apport en capital, mais en nombre de personnes représentées, pour conforter le modèle et éviter les dérives. Ainsi, le collège " producteurs » est dans un rapport de 1 à 100 par rapport au collège " consommateurs". Il détient pourtant $20 \%$ des droits de vote et bénéficie de fait d'une sur-représentation par rapport au nombre de sociétaires. A terme, cette répartition, tout comme la teneur des collèges, est susceptible d'évoluer pour rester en phase avec le développement de l'entreprise.

\section{LE DÉVELOPPEMENT, CLÉ DE LA RÉUSSITE ÉCONOMIQUE}

La réussite économique de ce projet, ou au moins la preuve de sa faisabilité et de sa pérennité, pouvait tenir de la gageure. L'ambition de fournir un service énergétique citoyen complet est complexe et s'inscrit directement dans une mission de service public et d'intérêt général.

Ce service énergétique étant une coopérative, celle-ci est ouverte à toute personne désirant s'y associer: c'est le principe de la " porte ouverte ». Cela veut dire aussi que seuls les membres participent à l'élaboration, à la gestion, à l'orientation de l'entreprise, dans l'idée de satisfaire leur intérêt collectif. Pourtant, nous pouvons aussi espérer (et œuvrer en ce sens) que la portée de ce travail soit d’intérêt général et puisse bénéficier à tous. 
Pour atteindre cet objectif, un grand nombre de sociétaires, une audience forte est nécessaire. D’autant plus que le domaine de l'énergie, en particulier celui de l'électricité, demande de gros moyens financiers et d'expertise. Enercoop est aujourd'hui la Scic la plus importante de France en nombre de sociétaires (environ 18000 en additionnant les dix coopératives) et en nombre de clients (environ 25000 fin 2015). Cela induit deux questions: faut-il encourager tous les clients à devenir sociétaires? Y a-t-il une limite au nombre de sociétaires?

La première question est en débat. Le propre de la Scic est bien d'avoir des bénéficiaires non sociétaires, la loi prévoyant que des relations commerciales avec les tiers sont possibles (art. 19 sexies). C'est peut-être là que se situe la relation de l'intérêt collectif qui se déploie en intérêt général. Un groupe « réduit » devient force de proposition pour le plus grand nombre. On peut effectivement penser que le produit est bon, mais sans pour autant vouloir participer à son élaboration, au sens large. Faut-il pousser un maximum de bénéficiaires à devenir sociétaires, ce qui aurait pour effet de renforcer le capital de la société? Ce renforcement ne comporte-t-il pas le risque de voir se constituer diverses catégories de sociétaires (sociétaires actifs, sociétaires soutiens, sociétaires fantômes qui ne prennent jamais part aux assemblées générales), comme c'est le cas aujourd'hui dans certaines banques ou mutuelles? Néanmoins, dans la phase actuelle de développement, l'accroissement du nombre de sociétaires donne du poids à l'action tout en fortifiant le capital. C'est une consolidation de la visibilité dont on ne peut se passer dans le contexte présent.

Ces observations renvoient à la seconde question: quelles limites, si elles existent? Avec pour corollaire: comment animer le sociétariat? La volonté d'Enercoop de construire avec et pour ses bénéficiaires place le sociétaire au centre des attentions et, sur ce point, la charte du réseau Enercoop est précise: "Animer la vie coopérative par la mise en place de dispositifs permettant l'expression et la prise en compte de la parole de tous les sociétaires ainsi que leur participation active.»C'est une partie intrinsèque des valeurs de la coopérative. Là aussi, la question n'est pas tranchée de savoir s'il existe un seuil à ne pas dépasser: est-ce le nombre de sociétaires, le nombre de salariés? Pourtant, pour bien souligner la prégnance de cette question, sans imposer un cadre strict, un accord a été trouvé pour inscrire dans la charte du réseau la nécessité de " conserver une taille humaine » et un contact direct avec les sociétaires.

\section{CONSTRUIRE UN RÉSEAU, CONSERVER SES VALEURS}

L'écoute des sociétaires et la volonté de relocaliser la gestion de l'énergie supposent une attention particulièrement vigilante. Ces objectifs orientent fortement l'action, en se renforçant mutuellement. Depuis 2009, pas moins de neuf autres Scic, portant elles aussi le nom d'Enercoop, se sont déployées sur le territoire métropolitain. Ce déploiement permet d'être à proximité des sociétaires et de s'adapter au mieux aux spécificités des territoires. Pierre Rosanvallon appelait à "la mise en œuvre de systèmes plus décentralisés [...] directement portés par des rapports sociaux concrets " (Rosanvallon, 1984, p.124). Cette décentralisation par duplication est en cours. 
Si la coopérative historique détient toujours certaines prérogatives, l'objectif est de rapprocher le sociétaire du lieu de décision, dans une dynamique citoyenne. L'objectif d'Enercoop est de montrer la faisabilité et la pertinence du modèle: énergies renouvelables et appropriation citoyenne pour réussir la transition énergétique pour tous.

\section{La dynamique du statut Scic}

Dans l'idée d'un service rendu aux citoyens, et donc de dépassement du seul intérêt collectif, le statut permet d'investir d'autres domaines où son utilité sociale et la qualité de ses sociétaires pourraient permettre une approche différente.

\section{DANS LE DOMAINE DE L'ÉDUCATION}

A côté du secteur public ou d'émanation confessionnelle, il existe des écoles associatives laïques. Elles émanent généralement d'un groupe identifié et moteur, qui se rassemble autour d'un projet pédagogique. Principalement présentes dans le domaine de l'école maternelle et élémentaire, elles peuvent être sous contrat (respect des programmes avec en contrepartie octroi de moyens), comme les écoles Freinet, Diwan ou Calandreta, ou hors contrat, comme les écoles Montessori ou Steiner. Ces écoles s’appuient généralement sur le statut associatif et une gouvernance relativement pyramidale. Le statut Scic pourrait être judicieusement adapté à ces projets. Il permettrait une véritable dynamique de répartition des pouvoirs et de construction de projets.

Dans certains établissements, par exemple dans les Calandretas, il existe déjà deux collèges de vote dans chaque école: l'équipe pédagogique, d'une part, et l'association (parents d'élèves et personnes impliquées dans le projet), d'autre part. Passer en Scic pourrait permettre l'expression de catégories d'associés de manière formelle. Tout en maintenant la catégorie des enseignants et celle des parents d'élèves, pourraient s'ajouter les personnels non enseignants, les institutions publiques (commune, région) comme les structures privées (partenaires culturels, instituts de formation, etc.) et même s'associer les élèves.

La Scic Conflent Energie intègre dans ses statuts un collège « Jeunes

(7) Voir les statuts de la Scic Conflent Energie, articles 12.2 et 18.1: Conflentenergie. free.fr/DOCS/SCIC $\% 20$ Conflent $\% 20$ Energie $\% 20$ -\%20Statuts-05-12-2013.pdf. (8) Voir la charte des Calandretas, article 3.5 Calandreta. org/IMG/pdf/charte_fr.pdf. sociétaires " ${ }^{(7)}$, défini comme " les bénéficiaires mineurs des activités de la Scic, représentés par leurs représentants légaux». Dans les Calandretas, des institutions dans les classes donnent la parole aux enfants ${ }^{(8)}$. Néanmoins l'idée développée par la Scic Conflent Energie consiste à associer à part entière tous les bénéficiaires. Naturellement, des garde-fous doivent être mis en place pour éviter la démagogie et la manipulation, mais le statut Scic permet cette appropriation par chacun du projet collectif auquel il participe.

\section{DANS LE DOMAINE DE LA SANTÉ}

La loi du 2 janvier 2002 rénovant l'action sociale et médico-sociale s'est attachée à développer et à encadrer le droit des usagers. Entre autres choses, elle instaure formellement le conseil de la vie sociale (CVS) ou une autre forme de participation pour que les bénéficiaires des prestations puissent 
participer au fonctionnement des établissements ${ }^{(9)}$. Pourtant, là aussi le modèle de la Scic pourrait être efficient, notamment par le biais de la santé communautaire, notion nouvelle apparue en France dans les années 90 .

La santé communautaire est le processus par lequel les membres d'une collectivité, géographique ou sociale, conscients de leur appartenance à un même groupe, réfléchissent en commun sur les problèmes de leur santé, expriment leurs besoins prioritaires et participent activement à la mise en place, au déroulement et à l'évaluation des activités les plus aptes à répondre à ces priorités.

Outre le domaine des addictions, celui des affections de longue durée ou celui de la gériatrie pourraient trouver des réponses adaptées, une appropriation de leur devenir plus prégnante à travers le statut Scic. Des actions comme l'autosupport (par exemple ASUD ${ }^{(10)}$ ), l'éducation thérapeutique (soumise à autorisation par les agences régionales de santé) ou les maisons de retraite (voir l'exemple de la Maison des Babayagas) pourraient opter pour le statut Scic afin de s'élargir à une communauté plus large: celle des citoyens.

La force du statut Scic, qui associe à parts égales plusieurs catégories d'associés, a bien pour vocation, dans certains cas, de s'élargir à une audience plus large. Son objet, qui vise à «la production et la fourniture de biens et de services qui présentent un caractère d'utilité sociale », se trouve alors à la lisière du secteur marchand.

\section{Conclusion}

Pour revenir à notre assertion liminaire, le statut Scic permet-il de remplacer ou de compléter un service public? Quelles sont les convergences? Comment les rapprocher? Le service public concerne tout le monde, l'un de ses échecs tient à la concentration du pouvoir, à la rigidité de ses orientations qui ont fait que, peu à peu, ses agents comme ses usagers se sont sentis à la fois déresponsabilisés et oubliés. La gouvernance de la Scic intègre toutes ces personnes dans la prise de décision, l'orientation et le suivi. Certes, la Scic diffère du service public par son mode de financement, puisque seuls les sociétaires y participent volontairement, alors que nous sommes tous contributeurs des missions du service public par le biais de l'impôt.

En 1931, Charles Gide décrivait une forme d'entreprise ayant pour objet des missions de service public: "Création d'entreprises à forme mixte. L'Etat ou la ville concède l'entreprise à une société par actions dont l'administration est tripartite, c'est-à-dire composée des représentants: a) de l'Etat (ou de la ville); b) des travailleurs; $c$ ) des consommateurs (ou, comme on dit plutôt quand il ne s'agit pas de consommations alimentaires ou vestimentaires mais d'utilisation de certains services, des usagers). On s'efforce d'associer ainsi tous les intérêts en jeu» (Gide, 1931, p. 156). Associer tous les intérêts en jeu, construire ensemble un intérêt collectif qui puisse être utile à l'intérêt général par l'association intelligente et pragmatique des intérêts individuels de citoyens: on arrive alors bien au-delà d'une simple somme de quelques individualismes, et c'est bien l'un des enjeux de la Scic que Gide décrivait avec soixante-dix ans d'avance. 
Quels sont les atouts de la Scic? Mettre autour de la table de la gouvernance toutes les parties prenantes. Tous les sujets, tous les projets sont envisageables. La pertinence de la Scic tient au fait que ce projet va être travaillé par un groupe dont le premier objectif sera d'identifier, de comprendre, d'imaginer, de récolter des idées, des points de vue pour alimenter une réflexion en profondeur qui se perpétuera dans la gouvernance. Ce vrai travail d'éducation populaire et partagé est l'un des fondements de la Scic.

A l'inverse, quels seraient les risques inhérents à la création une Scic? L'un d'eux pourrait paradoxalement être lié à son succès et à l'éloignement des sociétaires "de base ». Une grande attention doit être portée au maintien de cette démocratie de proximité, à l'écoute de tous, même de ceux qui ne sont pas fortement impliqués dans le fonctionnement de l'entreprise. De nombreuses questions se posent sur la limite d'expansion des Scic: limite géographique, nombre de sociétaires, nombre de salariés. Sans qu'il soit possible d'apporter des réponses précises sur ces limites, un facteur de limitation du risque de dispersion tient à l'autonomie de chaque coopérative, le réseau permettant le partage des valeurs et la mutualisation de certaines fonctions.

La Scic ne remplacera pas le service public dans toutes ses variétés, mais elle peut permettre une évolution de celui-ci par une réappropriation par chacun des enjeux, de la réflexion, de l'adaptation au territoire. Les services publics actuels sont perçus comme centralisateurs et technocratiques dans leurs orientations et leur mode de fonctionnement, alors qu'il existe un fort désir de proximité, de relocalisation, de participation. "Cette exigence de démocratie directe ne doit pas être négligée: elle ouvre en effet la possibilité d'instituer des services communs à l'échelle locale, lesquels pourraient former réseau et, en impliquant la population dans la construction des politiques, redonner sens à la citoyenneté politique et sociale» (Dardot, Laval, 2014). Pour investir cette dimension d'investissement citoyen, cette répartition de l'examen et de la prise de décision, la Scic est certainement un outil efficace pour passer, dans certains cas, du service public au service citoyen. 


\section{BibliogRAPHIE}

Becker A., Daune M., Guéville C. et al., 2015, « De la démocratie sociale à la gouvernance », in « Dossier Sécurité sociale», Pour, $\mathrm{n}^{\circ} 186, \mathrm{p} .21$.

Bezançon X., 1995, Les services publics en France du Moyen Age à la Révolution, Paris, Ecole nationale des ponts et chaussées.

Chevallier J., 2012 [1987], Le service public, coll. Que sais-je?, Paris, Presse universitaires de France.

Cohen A., 2008, « Futurologie, décroissance et utopisme: quelques mises en garde de la bioéconomie », Ecologie et Politique 2008/3 (n³7), p. 79-86.
Dardot P., Laval Ch., 2014, Commun, essai sur la révolution au $X X I^{e}$ siècle, Paris, La Découverte, p. 127-131 et 522.

Gide Ch., 1931, Principes d'économie politique, Paris, Librairie du recueil Sirey, p. 156.

Lavergne B., 1926, L'ordre coopératif, Paris, librairie Félix Alcan.

Rosanvallon P., 1984, La crise de l'Etatprovidence, Paris, Le Seuil.

Rossinot A., 1994, L'avenir de la fonction publique: une fonction publique est-elle encore utile à l'aube du $X X^{e}$ siècle?, Paris, ministère de la Fonction publique. 